\title{
Percutaneous endoscopic necrosectomy using a fully covered self-expandable metal stent in severe necrotizing pancreatitis
}
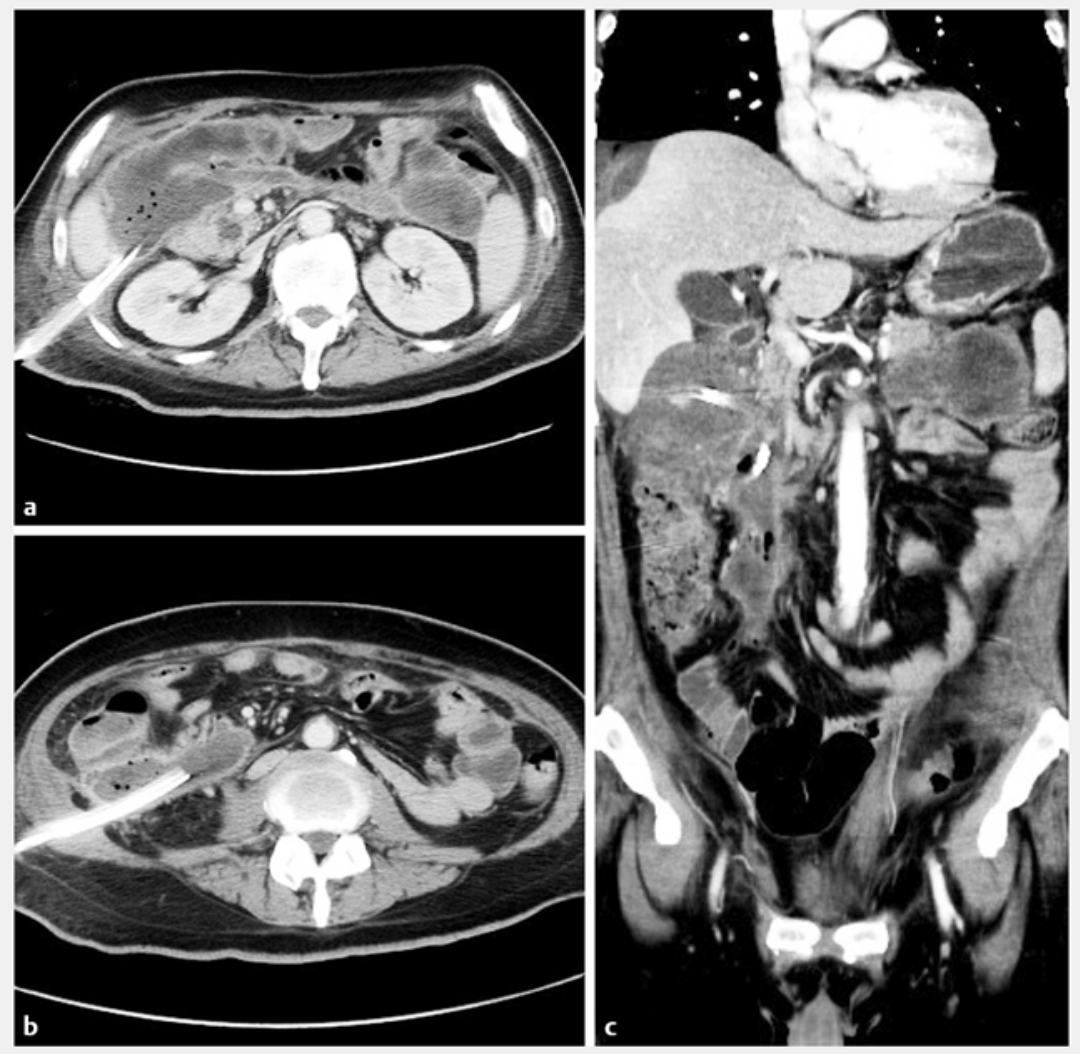

- Fig. 1 Contrast-enhanced abdominal computed tomography before treatment. a Previous subhepatic percutaneous drainage tube. b Previous percutaneous drainage tube in the right infrarenal space. c Coronal plane.

Direct endoscopic necrosectomy (DEN) is a minimally invasive treatment option that has been introduced in recent years for the treatment of infected walled-off pancreatic necrosis (WON), and has been performed as an alternative to operative and percutaneous therapy [1-3]. An esophageal, fully covered, self-expandable, metal stent (FCSEMS) has a large diameter and can provide effective drainage for pancreatic collections. Both ends are flared, allowing apposition, preventing migration, and facilitating endoscope passage and debridement in the retroflexed position. Herein, we demonstrate technically and clinically successful treat- ment of WON by DEN using an FCSEMS in a patient who was not a candidate for a transgastric approach. This treatment could be a good therapeutic option for infected WON lesions located some distance from the gastric wall.

A 48-year-old man presented with infected pancreatic and peripancreatic necrosis 4 weeks after the onset of severe necrotizing pancreatitis. Computed tomography (CT) scan revealed a large well demarcated WON involving the subhepatic space, which extended downward to the right colonic mesenteric space and included a $30-\mathrm{mm}$ partially solid necrosis at the pancreatic tail. Although the pa-

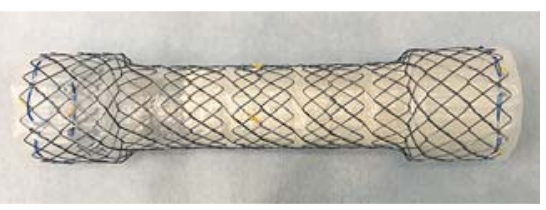

- Fig. 2 An esophageal, fully covered, self-expandable, metal stent (Hanarostent, diameter $22 \mathrm{~mm}$, length $90 \mathrm{~mm}$; M.I. Tech., Seoul, Korea).

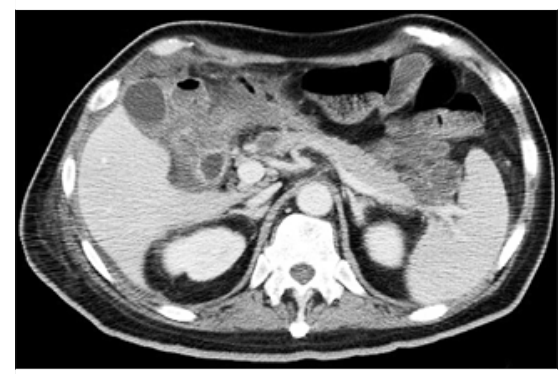

- Fig. 3 Follow-up contrast-enhanced abdominal computed tomography after four sessions of direct endoscopic necrosectomy.

tient was treated with intravenous antibiotics and percutaneous drainage of the subhepatic and right infrarenal space, only the liquid drained away and the solid necrotic matter remained ( $\triangleright$ Fig. 1 ). Therefore, the patient was referred to our hospital for treatment of the infected pancreatic necrosis.

First, the necrotic cavity was accessed by advancing a 0.025 -inch guidewire through the previous infrarenal percutaneous drainage tube under fluoroscopic guidance. The FCSEMS (Hanarostent, diameter $22 \mathrm{~mm}$, length $90 \mathrm{~mm}$; M.I. Tech, Seoul, Korea) ( $>$ Fig. 2 ) was passed over the guidewire and into the intra-abdominal cavity under fluoroscopic guidance, which also confirmed cavity access. The stent was successfully deployed in the necrotic cavity by simple retraction of the catheter sheath.

In the second step, we performed DEN using a forward-viewing therapeutic gas- 


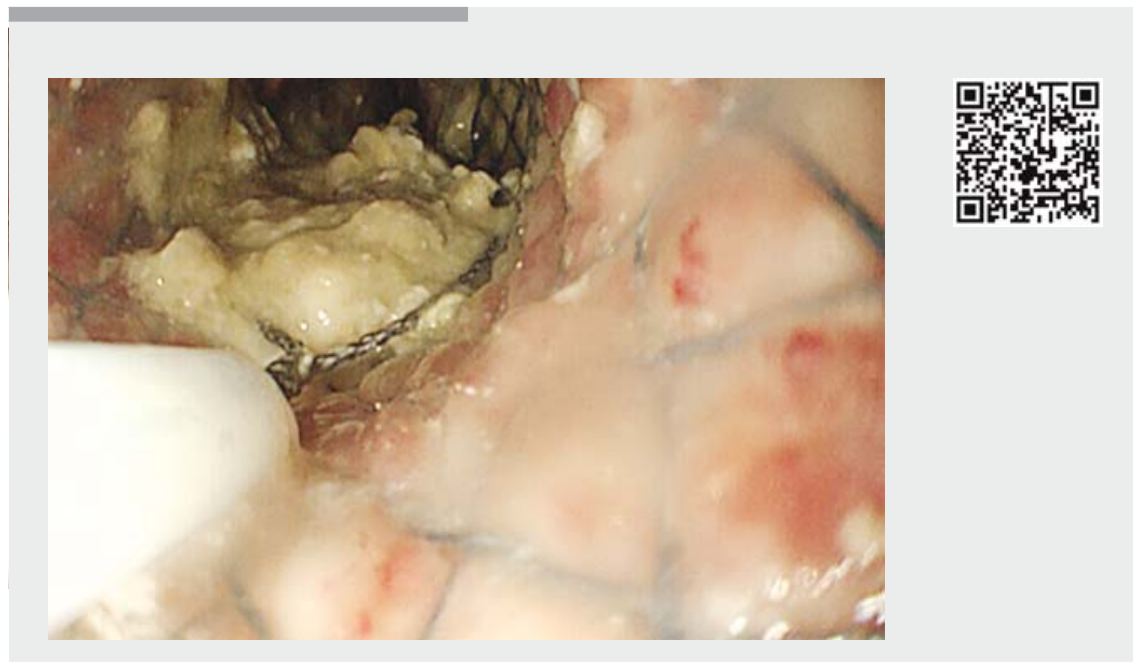

Video 1 Percutaneous endoscopic necrosectomy using a fully covered, self-expandable, metal stent in severe necrotizing pancreatitis.

troscope via the FCSEMS route. The snare catheter was introduced to perform repeated mechanical debridement. To avoid bleeding and injury to the intact tissue, we slowly grasped the superficial layers and pulled without significant resistance. The necrotic tissue culture showed Corynebacterium striatum infection.

The procedure was repeated every $2-3$ days until the necrotic material was completely removed and a clean cavity, lined with granulation tissue, was achieved. Between the necrosectomy sessions, the FCSEMS remained in place and was finally removed at the end of last debridement session. The patient required a total of four endoscopic debridement sessions in order to almost completely remove the necrotic debris. He subsequently made a rapid recovery.

Follow-up CT documented a dramatic decrease in the size of the WON and a small lesion at the pancreatic tail, which could be resolved by medication alone ( $>$ Fig. 3, $>$ Video 1).

Endoscopy_UCTN_Code_TTT_1AR_2AI

\section{Competing interests}

None

The authors

Issaree Laopeamthong', Ryosuke Tonozuka ${ }^{2}$, Hiroyuki Kojima², Shuntaro Mukai²,

Takayoshi Tsuchiya², Atsushi Sofuni², Takao Itoi $^{2}$

1 Department of Surgery, Bangkok Metropolitan Administration Medical College and Vajira Hospital,

Navamindaradhiraj University, Bangkok, Thailand

2 Department of Gastroenterology, Tokyo Medical University Hospital, Shinjuku, Tokyo, Japan

\section{Corresponding author}

\section{Ryosuke Tonozuka, MD}

Department of Gastroenterology and Hepatology, Tokyo Medical University, 6-7-1 Nishishinjuku, Shinjuku-ku, Tokyo 160-0023, Japan

Fax: +81-3-53816654

tonozuka1978@gmail.com

[1] Seifert H, Wehrmann T, Schmitt T et al. Retroperitoneal endoscopic debridement for infected peripancreatic necrosis. Lancet 2000; 356: 653-655

[2] Seewald S, Groth S, Omar S et al. Aggressive endoscopic therapy for pancreatic necrosis and pancreatic abscess: a new safe and effective treatment algorithm. Gastrointest Endosc 2005; 62: $92-100$

[3] Ang TL, Kwek AB, Tan SS et al. Direct endoscopic necrosectomy: a minimally invasive endoscopic technique for the treatment of infected walled-off pancreatic necrosis and infected pseudocysts with solid debris. Singapore Med J 2013; 54: 206 - 211

\section{Bibliography}

DOI https://doi.org/10.1055/a-0756-8203

Published online: 23.11.2018

Endoscopy 2019; 51: E22-E23

(c) Georg Thieme Verlag KG

Stuttgart · New York

ISSN 0013-726X

\section{ENDOSCOPY E-VIDEOS}

https://eref.thieme.de/e-videos

口回 Endoscopy E-Videos is a free

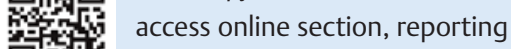
靣触: on interesting cases and new

techniques in gastroenterological endoscopy. All papers include a high quality video and all contributions are freely accessible online.

This section has its own submission website at https://mc.manuscriptcentral.com/e-videos 